\title{
Health services provision of 48 public tertiary dental hospitals during the COVID-19 epidemic in China
}

\author{
Yang Yang ${ }^{1} \cdot$ Yin Zhou ${ }^{2} \cdot$ Xiaoqiang Liu ${ }^{1} \cdot$ Jianguo $\operatorname{Tan}^{1}$
}

Received: 25 February 2020 / Accepted: 23 March 2020 / Published online: 3 April 2020

(C) Springer-Verlag GmbH Germany, part of Springer Nature 2020

\begin{abstract}
Objectives To assess the status of health services provision of public tertiary dental hospitals during the COVID-19 epidemic in China and to evaluate the regional difference of telehealth.

Materials and methods The health services provision of public tertiary dental hospitals in China mainland during the COVID-19 epidemic was inquired. The status of non-emergency dental services, emergency dental services, and online professional consultation and the hospitals' geographical distribution were recorded and analyzed.

Results All the 48 public tertiary dental hospitals suspended general non-emergency dental treatment while providing emergency dental services only. Ninety percent of them notified the change of dental services online, and $69 \%$ of them offered free online professional consultations. The penetration rate of online technology was significantly higher in the eastern region than that of the central and western regions.

Conclusions There was a significant change in the health service provision of Chinese public tertiary dental hospitals during the COVID-19 epidemic and wider use of telehealth in the eastern region.

Clinical relevance This report demonstrated that dental health services were significantly affected by the COVID-19 epidemic in China, which might lead to a long-time impact on dental care in the future.
\end{abstract}

Keywords COVID-19 $\cdot$ Epidemics $\cdot$ Dental care $\cdot$ Telehealth

\section{Introduction}

In December 2019, the COVID-19 outbreak was first reported from Wuhan, Hubei, China [1]. The novel coronavirus, the severe acute respiratory syndrome coronavirus 2 (SARS$\mathrm{CoV}-2$ ), rapidly transmitted to all provinces (autonomous regions and municipalities) of China and dozens of other countries, and has raised widespread national and international

Yang Yang and Yin Zhou contributed equally to this work.

Xiaoqiang Liu

liuxiaoqiang@bjmu.edu.cn

1 Department of Prosthodontics, Peking University School and Hospital of Stomatology \& National Clinical Research Center for Oral Diseases \& National Engineering Laboratory for Digital and Material Technology of Stomatology \& Beijing Key Laboratory of Digital Stomatology, 22 Zhongguancun Avenue South, Haidian District, Beijing 100081, People's Republic of China

2 Department of Anesthesiology, Peking University First Hospital, Beijing 100034, People's Republic of China concerns. Although the authorities have taken proactive measures to combat COVID-19, the case count from the SARSCoV-2 soared continually. Until 22 February 2020 , there were 78,811 confirmed cases globally with 2462 (3.1\%) deaths in 29 countries [2].

As its outbreak unfolds, prevention and control of COVID19 have become a major concern, there of the transmission routes continually receiving much research attention recently. According to the guidelines on diagnosis and treatment of the COVID-19 (6th trial edition) from China National Health Commission [3], respiratory droplets and close contact are the main routes of transmission. Meanwhile, prolonged exposure to high concentrations of aerosol in a relatively closed environment is a risk of aerosol transmission [3]. The literature showed that many dental procedures produce aerosols and droplets that are contaminated with bacteria, viruses, and blood and have the potential to spread such infections to dental personnel and other people in the dental office [4]. Consequently, according to the Chinese authorities' recommendations and with fears of COVID-19, people are reluctant to go outside but stay in house. Our previous investigation 
showed that patients were less willing to go to dental institutions, and the proportion of dental and oral infection in emergency dental care rose at the beginning of the epidemic [5].

The public tertiary dental hospitals in China always play a key role in dental health services. Therefore, a critical challenge for them is to prevent nosocomial COVID-19 transmission and provide health services for the general population. In this study, we aimed to assess the current status of health services provision of public tertiary dental hospitals during the COVID-19 epidemic in China. Furthermore, as the eastern cities and central/western cities have different social development levels, the regional difference was also evaluated.

\section{Methods}

We retrieved the public tertiary dental hospitals in China mainland through a web search engine and all of them were involved in the present report. Their health services provision was inquired via official websites, official accounts in social software WeChat, and official telephones. The following information was recorded: whether non-emergency dental treatments, emergency dental services, and online professional consultation were available. If the telehealth was provided, the charge, access way, service time, and service content were further investigated. Hospitals' geographical distribution was recorded and classified as the eastern region or the central and western regions of China (Fig. 1). The retrieve period during the COVID-19 epidemic was between 17 February and 23 February 2020.

Categorical variables are presented as $N(\%)$ and analyzed through SPSS Statistics, version 20.0 (IBM Corp., Armonk, NY, USA) using chi-square test. The significance level was set at 0.05 .

\section{Results}

There were 48 public tertiary dental hospitals in China mainland enrolled in the present report. These hospitals were distributed in 35 cities, with 1 to 3 hospitals in each city. Their health services provision changed significantly (Table 1). All the hospitals suspended general non-emergency dental treatment while providing emergency dental services for acute toothaches, dental trauma, oral and maxillofacial trauma, and cellulitis and abscess lesions. Nine-tenths of them notified the change of dental services via a web-based and/or mobilebased network, while the others provided on-site or passive telephone notification only. As the usual face-to-face nonemergency dental services were canceled, $69 \%$ of the hospitals offered free online professional consultations for determining whether a condition was necessary for emergency treatment, and providing home dental and oral care guidance.
The vast majority of those services were easily accessible through a widely used social software called WeChat. About two-fifths of the telehealth was $24 \mathrm{~h}$ of continuous service, while the others were time-limited.

Of all the 48 hospitals, 23 were in the eastern region and 25 were in the central and western regions (Table 2). Regardless of locations, there was no significant difference in the utilization of a mobile-based notification system $(P=0.187)$. Broader usage of web-based notice was observed in the hospitals in the eastern region $(P=0.031)$. In terms of the online professional consultation, the penetration rate was significantly higher in the eastern region than that of the central and western regions $(87.0 \%$ vs. $52 \%)(P=0.009)$.

\section{Discussion}

This report presented meaningful findings of the current status of health services provision of 48 public tertiary dental hospitals, and the difference between the eastern and central/ western regions in China mainland. The results strongly suggested that the COVID-19 epidemic significantly affected the dental hospitals' health services. The public tertiary dental hospitals received a large majority of dental patients in China, not merely because of the dental insurance but also the abundant dental resources and experts in public hospitals. They were the most representative in the Chinese dental health services. Although limited, this present report of 48 public tertiary dental hospitals reflected the current situation of the majority of public dental institutions.

Infection control is always the cornerstone of safe dental practice, especially during the COVID-19 epidemic. There would be a potential risk for transmission of acute viral respiratory tract infections during dental procedures because of aerosol production [6]. Thus, according to the guidelines of the Chinese Stomatological Association (CSA) [7], suspending general non-emergency dental treatment has potential importance in the prevention and control of COVID19. However, it may have a negative impact on patients' dental health or treatment effects, such as the prolonged orthodontic treatment time. Patients' psychological status (e.g., fear of infection) would also affect the progression of oral mucosa diseases [8]. There have been online questionnaires supplied by some public tertiary dental hospitals to identify whether their dental patients were anxious or worried about the delayed dental visit. Although the public dental hospitals still offered emergency treatments for patients, significant utilization reduction in emergency dental services was observed at the beginning of the COVID-19 epidemic [5]. Some dental specialists were also concerned about exploding visits after the end of the epidemic. The strengths of administrative departments of the government are suggested to be coordinated to implement comprehensive prevention and control measures 


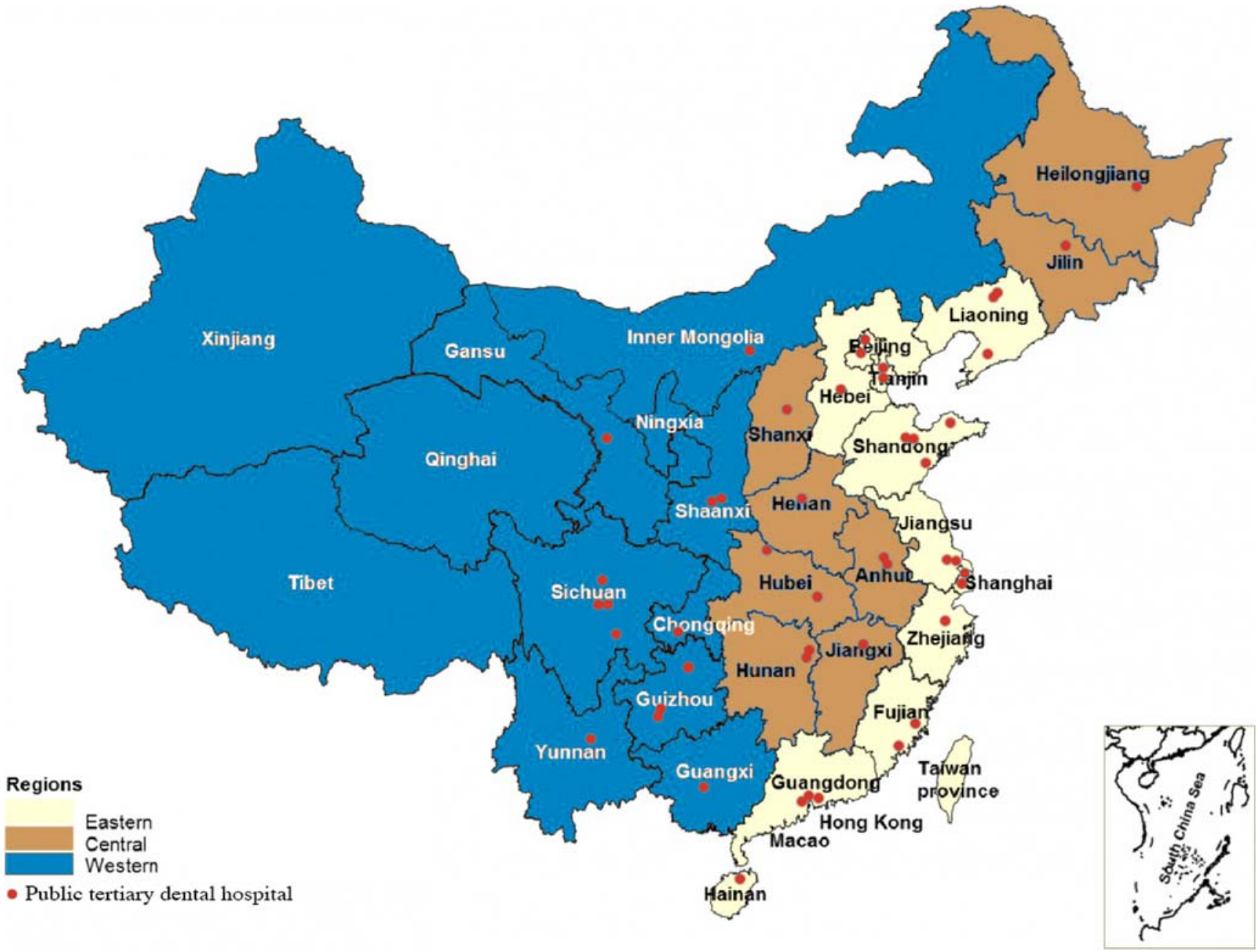

Fig. 1 Hospitals'geographical distribution and categorization. The red point represented 48 public tertiary dental hospitals in China mainland

in future dental care. However, there should be further studies about the long-term socioeconomic, psychological, or physical impacts of the current provision of dental services during the COVID-19 epidemic.
Table 1 Status of the provision of health services by 48 public tertiary dental hospitals during the COVID-19 epidemic

\begin{tabular}{lll}
\hline Theme & & $n(\%)$ \\
\hline Total number of hospitals & & 48 \\
Cities distributed & & 35 \\
Hospitals suspended non-emergency dental treatment & & $48(100 \%)$ \\
Hospitals provided emergency dental services & & $48(100 \%)$ \\
Measures of notifying the changes in health service & Mobile-based notice & $43(89.6 \%)$ \\
& Web-based notice & $30(62.5 \%)$ \\
& None of the above & $5(10.4 \%)$ \\
Hospitals provided online professional consultation & & $33(68.8 \%)$ \\
Charge & Free & $33(100 \%)^{*}$ \\
Access way & Via WeChat & $31(93.9 \%)^{*}$ \\
Service time & Via another app & $2(6.1 \%)^{*}$ \\
& 24 h & $13(39.4 \%)^{*}$ \\
& Time-limited & $20(60.6 \%)^{*}$ \\
\hline
\end{tabular}

*\% of hospitals providing online professional consultation $(n=33)$ 
Table 2 Online notification measures and online professional consultation of 48 tertiary dental hospitals in the eastern region and central and western regions $(n(\%))$

\begin{tabular}{|c|c|c|c|c|c|c|c|}
\hline & \multirow[t]{2}{*}{ Number of hospitals } & \multicolumn{2}{|c|}{ Mobile-based notice } & \multicolumn{2}{|c|}{ Web-based notice } & \multicolumn{2}{|c|}{ Professional consultation } \\
\hline & & Yes & No & Yes & No & Yes & No \\
\hline Eastern region & 23 & $22(95.7 \%)$ & $1(4.3 \%)$ & $18(78.3 \%)$ & $5(21.7 \%)$ & $20(87.0 \%)$ & $3(13.0 \%)$ \\
\hline Central and western region & 25 & $21(84.0 \%)$ & $4(16.0 \%)$ & $12(48.0 \%)$ & $13(52.0 \%)$ & $13(52.0 \%)$ & $12(48.0 \%)$ \\
\hline$P$ value & - & 0.187 & & 0.031 & & 0.009 & \\
\hline
\end{tabular}

In this report, the supply of online dental consultation was significantly different between hospitals in different regions. The penetration rate was significantly higher in the eastern region than that of central/western regions (87.0\% vs. $52.0 \%$ ), which might be related to the economic development and oral health status. The online consultation, being a subset of teledentistry, provides education and preventive care to dental patients and has the potential to identify high-risk populations [9]. It is an innovative method of oral health service delivery that can connect dental practitioners and patients. This is important particularly for underserved oral care due to the COVID-19 epidemic or other reasons like geographical barriers or dental workforce shortages. The efficacy and benefits of online dental consultation have supported it integrating into routine dental health service [10], and the wide application during the epidemic would help its extensive promotion. The development of consultation software or apps was desired not only by patients but also by medical specialists, for better data consolidation, to access patients' previous medical records and facilitate their referrals to dental hospitals or clinics. The encouragement of online dental consultation should be enhanced especially for rural and remote areas in central and western regions to improve their locally based medical care.

Within the limitation of this report, we observed significant changes in the health service provision of Chinese public tertiary hospitals during the COVID-19 epidemic. All the public tertiary dental hospitals suspended general non-emergency dental treatment while providing emergency dental services only. More than two-thirds of them offered free online professional consultations. There was a significantly higher penetration rate in the utilization of telehealth in the eastern region than that in the central/western regions. Dental health services were significantly affected by the COVID-19 epidemic in China currently. However, further study should focus on the potential long-time impact the epidemic may lead to dental care.

Acknowledgments The authors paid tribute to all who contributed to the fight against COVID-19.

Funding information This work was supported by Peking University School and Hospital of Stomatology (PKUSSNCT-19A03).

\section{Compliance with ethical standards}

Conflict of interest The authors declare that they have no conflict of interest.

\section{References}

1. Li Q, Guan X, Wu P. et al (2020) Early transmission dynamics in Wuhan, China, of novel coronavirus-infected pneumonia. New Engl J Med 382:1199-1207. https://doi.org/10.1056/ NEJMoa2001316

2. WHO (2020) Coronavirus disease 2019 (COVID-19) situation report-34. World Health Organization web. https://www.who.int/ emergencies/diseases/novel-coronavirus-2019/situation-reports. Accessed 23 Feb 2020

3. China NHC (2020) Guidelines on diagnosis and treatment of the COVID-19 (6th trial edition). ChinaNHCWeb. http://www.nhc. gov.cn/xcs/fkdt/202002/54e1ad5c2aac45c19eb541799bf637e9. shtml. Accessed 19 Feb 2020

4. Harrel SK, Molinari J (2004) Aerosols and splatter in dentistry: a brief review of the literature and infection control implications. J Am Dent Assoc 135:429-437. https://doi.org/10.14219/jada. archive.2004.0207

5. Guo H, Zhou Y, Liu X, Tan J (2020) The impact of the COVID-19 epidemic on the utilization of emergency dental services. J Dent Sci. https://doi.org/10.1016/j.jds.2020.02.002

6. Jakubovics N, Greenwood M, Meechan JG (2014). General medicine and surgery for dental practitioners: part 4 . Infections and infection control. Br Dent J 217:73-77. https://doi.org/10.1038/sj. bdj.2014.593

7. Chinese Stomatological Association(2020). Guidelines of clinical dental care during the COVID-19 epidemic. Chinese Stomatological Association Web. http://www.cndent.com/ archives/66974. Accessed 2 Feb 2020

8. Lin L, Duan N, Wang X, Song Y, Wang W (2019) Study on the correlation between common diseases of oral mucosa and psychological factors. J Prev Treat Stomatol Dis 27:236-240. https://doi. org/10.12016/j.issn.2096-1456.2019.04.006

9. Estai M, Kanagasingam Y, Tennant M, Bunt S (2018) A systematic review of the research evidence for the benefits of teledentistry. $J$ Telemed Telecare 24:147-156. https://doi.org/10.1177/ $1357633 X 16689433$

10. Daniel SJ, Kumar S (2014) Teledentistry: a key component in access to care. J Evid Based Dent Pract 14(Suppl):201-208. https:// doi.org/10.1016/j.jebdp.2014.02.008

Publisher's note Springer Nature remains neutral with regard to jurisdictional claims in published maps and institutional affiliations. 